\title{
Anesthetic Approach of Pregnant Woman with Cerebral Arteriovenous Malformation and Subarachnoid Hemorrhage during Pregnancy: Case Report
}

\author{
Catarina Santos Carvalho* ${ }^{1}$, Filipa Resende ${ }^{1}$, Maria João Centeno ${ }^{2}$, Isabel Ribeiro ${ }^{3}$, \\ João Moreira 4
}

1. Resident Physician, Hospital Garcia de Orta (HGO) Almada, Portugal

2. Graduate Hospital Assistant, HGO, Almada, Portugal

3. Head of Service; Graduate Hospital Assistant, HGO, Almada, Portugal

4. Head of Service; Service Director, HGO, Almada, Portugal

Received from Hospital Garcia de Orta, Almada, Portugal.

Submitted on January 19, 2012. Approved on May 7, 2012.
Keywords:
Intracranial
Arteriovenous
malformation;
Cesarean Section;
Neurosurgery:
Subarachnoid
Hemorrhage.

\begin{abstract}
Background and objectives: Subarachnoid hemorrhage (SAH) during pregnancy is a rare event, and about half the cases are due to arteriovenous malformations (AVM). The authors describe the anesthetic approach of a 39 week pregnant patient scheduled for cesarean section, with a history of SAH due to AVM at 22 week gestation.

Case report: 39 week pregnant patient, healthy prior to pregnancy, with a history of SAH at 22 week gestation, manifested by headache, vomiting, and dizziness without loss of consciousness or other deficits on admission to the emergency room. Magnetic resonance imaging (MRI) revealed a left frontal AVM. After a short hospital stay for stabilization and diagnosis, the final medical decision was to maintain the pregnancy and a multidisciplinary follow-up by neurosurgery and high-risk obstetric consultation. An elective cesarean section was performed at 39 weeks under epidural anesthesia. During the intraoperative period, an episode of hypotension rapidly reversed with phenylephrine occurred. The newborn Apgar score was 10/10. An epidural catheter was used for postoperative analgesia, also uneventful.

Conclusions: There are very few published cases of anesthetic approach for pregnant women with symptomatic AVM. All decisions made by the multidisciplinary team, from choosing to continue the pregnancy to the ideal time for AVM intervention and type of anesthesia and analgesia, were weighted according to the risk of brain damage. Regarding the anesthetic procedure, the authors emphasize the need for hemodynamic stability.

๑ 2013 Sociedade Brasileira de Anestesiologia. Published by Elsevier Editora Ltda. All rights reserved.
\end{abstract}

*Corresponding author: E-mail: catarinascarvalho@gmail.com

ISSN/\$ - see front metter ๔ 2013 Sociedade Brasileira de Anestesiologia. Published by Elsevier Editora Ltda. All rights reserved. 


\section{Introduction}

Brain arteriovenous malformations (AVM) typically appear between the age of 25 and 45 years, with a peak incidence in the $4^{\text {th }}$ decade ${ }^{1-4}$. Despite its congenital origin, only $18-20 \%$ are diagnosed in childhood ${ }^{5}$.

AVM may present itself as intracranial hemorrhage (30$86 \%)$, seizures $(8-46 \%)$ and/or focal neurological deficits (4-23\%), headache (up to 15\%), hydrocephalus and, rarely, congestive heart failure ${ }^{4,6}$.

Incidence of subarachnoid hemorrhage (SAH) during pregnancy is estimated at about $1-10$ per 100,000 pregnant women ${ }^{7}$. AVM are responsible for about $50 \%$ of $\mathrm{SAH}$ in pregnant women and the $3^{\text {rd }}$ cause of non-obstetric maternal morbidity $^{8}$. However, it is known that even without rupture, AVM may cause brain damage by brain "steal phenomenon" or by decreasing the cerebral perfusion ${ }^{6}$.

There is controversy about the influence of pregnancy on natural history of AVM; however, the risk of first rupture during pregnancy is $3.5 \%$, which is similar to non-pregnant woman ${ }^{9}$. In these cases, treatment during pregnancy is based on neurological factors rather than obstetric indications ${ }^{3}$.

The authors describe the anesthetic strategy for a pregnant woman scheduled for elective cesarean section, with a history of SAH due to AVM rupture at 22 weeks of gestation. The approach, performed by a multidisciplinary team: (obstetrician, anesthesiologist, neurologist, and neonatologist) implied the following: clarification of the diagnosis and clinical stabilization of the baseline condition, decision to continue the pregnancy, follow-up during the remainder of pregnancy, safety of anesthesia and delivery, and AVM embolization in the postpartum period.

\section{Case Report}

Female patient, 30 years old, 39 weeks of gestation, obese $\left(B M I=31 \mathrm{~kg} \cdot \mathrm{m}^{-2}\right)$, nillipara, admitted for elective cesarean section. Patient received follow-up from the $22^{\text {nd }}$ gestational week in multidisciplinary consultation by SAH in the context of AVM.

At 22 weeks of gestation, the patient was admitted to the Emergency Service with acute occipital headache and persistent dizziness with 10 hours of evolution, without focal neurologic deficits or loss of consciousness. After fetal well-being was confirmed by obstetrics, the patient was admitted to the neurosurgery department for diagnostic and clinical stabilization.

Cranial Computed Axial Tomography (CAT-scan) showed minor bleeding of the right caudate nucleus and the fourth ventricle.

Cerebral magnetic resonance imaging (MRI) revealed cortical-ventricular AVM on left frontal region, with about 4 $\mathrm{cm}$ in diameter, with nutrition of the anterior cerebral artery and drainage to the superior longitudinal sinus, without evidence of previous bleeding or parenchymal ischemic lesion (Figures $1 \mathrm{~A}$ and $1 \mathrm{~B}$ ).

During the patient's hospital stay, there was a favorable clinical and radiological evolution without neurological deterioration; therefore, the patient and doctors decided



Figure 1A Sagital view.

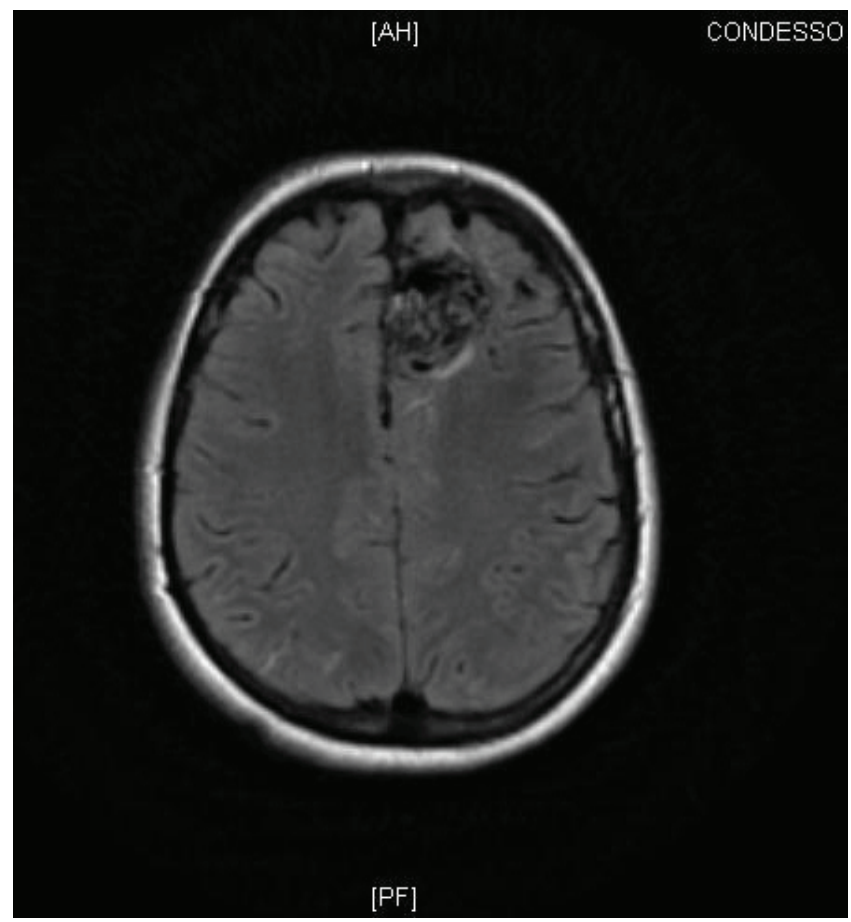

Figure 1B Axial view.

to continue pregnancy until fetal maturity. In this context, it was decided to postpone the diagnostic angiography and eventual embolization to postpartum period.

The patient then received regular follow-up with neurosurgery, obstetrics, and anesthesiology visits, presenting a stable condition without making use of any medicines. Although normal delivery was not contraindicated, the team proposed and discussed performing an elective cesarean section with the patient. 
On schedule, the patient was admitted for cesarean section, clinically asymptomatic from neurological and cardiovascular standpoints. In the delivery room, the team continuously monitored electrocardiographic tracing, pulse oximetry, invasive blood pressure, and respiratory rate, aside from assessing fetal well-being.

Cesarean section was performed under epidural anesthesia with titrated administration of $14 \mathrm{~mL}$ lidocaine (with epinephrine) at $20 \mathrm{mg} \cdot \mathrm{mL}^{-1}$ and sufentanil at 1 microg. $\mathrm{mL}^{-1}$ with sensory block ${ }^{\mathrm{T}}$.

During the intraoperative period, there was an isolated episode of hypotension (MBP drop not exceeding $20 \%$ of baseline), reversed with two intravenous bolus of phenylephrine (50 mg), without other complications. An intensive care unit (ICU) stay was prescheduled but it was not necessary.

After delivery, the team guaranteed hemostasis by uterine massage and slow infusion of oxytocin (40U in $1000 \mathrm{~mL}$ of $5 \%$ dextrose in saline, at about $80 \mathrm{~mL} \cdot \mathrm{h}^{-1}$ ), without hemodynamic repercussion.

The male child was born weighing $3,230 \mathrm{~g}$ with Apgar score of $10 / 10$ at 1 and 5 minutes, respectively.

Postoperative analgesia was maintained with $1 \mathrm{~g}$ of intravenous paracetamol every 6 hours and 3 epidural bolus of $10 \mathrm{~mL}$ ropivacaine $\left(1.2 \mathrm{mg} \cdot \mathrm{mL}^{-1}\right)$ and morphine $\left(0.2 \mathrm{mg} \cdot \mathrm{mL}^{-1}\right)$ every 12 hours (until 36 hours after cesarean section). For rescue analgesia, an epidural bolus of $6 \mathrm{~mL}$ ropivacaine (1.2 $\left.\mathrm{mg} \cdot \mathrm{mL}^{-1}\right)$ was prescribed whenever numerical pain scale $>4$ (respecting intervals between administrations over 1 hour). Pain at rest was always under control; pain upon mobilization was controlled by using two rescue boluses: and another at 6 and one at 18 hours post-cesarean. The parturient recovered bowel and bladder function spontaneously within 24 hours, without nausea or vomiting, itching or motor blockade during epidural analgesia.

In the immediate postpartum period, there were no complications from the obstetric or anesthetic standpoint. There were no hemodynamic changes nor neurological decompensation manifestations.

The patient was discharged on the fourth day postpartum, clinically stable and referred to the interventional neuroradiology consultation.

Eight weeks after delivery, AVM embolization was performed under deep sedation with remifentanil infusion without complications. The patient was discharged clinically asymptomatic at the sixth day post-embolization. Currently, she maintains follow-up with neurosurgery.

\section{Discussion}

There are few case reports and studies of AVM management during pregnancy, particularly those evaluating intracranial hemorrhage $(\mathrm{ICH})$ in this context ${ }^{10,11}$. This is a controversial topic even today $1,3,4,7,8,12$.

In 1974, Robinson et al., ${ }^{13}$ evaluating 24 cases, concluded that a woman who has had AVM would have $87 \%$ chance of developing ICH during pregnancy. On the other hand, Horton et al. ${ }^{9}$ in 1990, in a study of 451 women of childbearing age with AVM, concluded that pregnancy does not increase the risk of bleeding of an AVM, estimating it at 3.5\% (risk of bleeding in the non pregnant female population of childbearing age, with an AVM, estimated at 3.1\%). On the other hand, the risk of re-bleeding at one year after the first bleeding during pregnancy in women whose AVM was not intervened immediately after the event, appears to be increased to $6 \%$, compared to the risk of re-bleeding in non-pregnant population ${ }^{3,9}$.

The therapeutic strategy in these situations is based on case-by-case weighted evaluation of the treatment risks versus the risk of bleeding during the remainder of pregnancy, if AVM is not treated. Risks to the fetus should also be considered during the intervention, either by embolization, surgical, or other techniques. Although the best strategy for management of symptomatic AVM during pregnancy is not yet defined, one can say that the postponement of angiography to the postpartum period has the obvious advantage of minimizing fetal exposure to radiation and contrast medium, aside from avoiding the significant risk of complications during intervention $^{3,12}$. In most cases, the risk-benefit analysis seems not to support the elective treatment of AVM during pregnancy ${ }^{3}$.

In what concerns the delivery, despite what had been previously reported ${ }^{13}$, there is no evidence that normal delivery is associated with an increased bleeding risk in pregnant women with intact AVM. Horton et al. believe that delivery in a patient with AVM, which was not surgically excised, can be performed either vaginally or by cesarean section 3,9,14. On the other hand, there are no studies comparing delivery modes in pregnant women with AVM rupture (ICH), but in the few cases reported the option was to perform cesarean sections ${ }^{10,14}$.

In the case described here, elective cesarean section was the option of the multidisciplinary team, considering not only the risk of re-bleeding, the control of timing and duration of delivery and hemodynamic stability during childbirth. Reservation of neonate and adults ICU vacancies were taken into account on the planning.

From a strictly anesthetic point of view in the absence of established guidelines, our primary goal was to maintain parturient cardiovascular stability ${ }^{10}$, avoiding hypotension, (which could compromise the uteroplacental vascularization), and hypertension, (which could increase the risk of bleeding). In this sense, we discuss two dichotomies: general anesthesia / neuraxial anesthesia and epidural anesthesia/ spinal anesthesia.

Although the technique of general anesthesia (GA) with opioids, propofol, and volatile anesthetics is described as safe in emergencies in these pregnant women ${ }^{10}$, neuraxial anesthesia has indisputable advantages over hemodynamic instability caused by endotracheal intubation and extubation, and avoids increased intracranial pressure associated with positive pressure ventilation and emesis associated with GA.

Comparing epidural and spinal block, in the first, the more gradual onset of sympathetic blockade and existence of compensating phenomena seem to favor hemodynamic stability ${ }^{15}$.

The local anesthetic and adrenalin absorption through the epidural space appears to have an effect with increased cardiovascular output and heart rate ${ }^{16}$.

We chose to perform an epidural blockade with fractionated administration of lidocaine with epinephrine (20 $\mathrm{mg} \cdot \mathrm{mL}^{-1}$ ), with a total of $14 \mathrm{~mL}$. We emphasize that the anesthetic option was discussed and defined together with the neurosurgeon. Lidocaine with epinephrine was the lo- 
cal anesthetic chosen since it is the most frequently in our institution. The available studies seem to show no benefit of a local anesthetic over another ${ }^{16}$.

The patient was monitored before anesthesia with invasive pressure for a more reliable control of hemodynamic changes. The episode of hypotension was rapidly and easily reversed with phenylephrine, a $a_{1}$ agonist; evidence shows that phenylephrine seems to be more appropriate for hypotension after regional anesthesia in pregnant women compared to other vasopressors ${ }^{17}$.

\section{Conclusion}

Patients with symptomatic AVM during pregnancy are challenging in terms of medical approach in general, and of anesthetic management in particular. There is no consensus regarding the optimal timing for AVM treatment or for delivery, as well as for the most appropriate delivery method and anesthesia technique.

In the case described here, pregnancy seemed viable to the medical team involved. A 39-week pregnant woman had a close monitoring and the cesarean section was performed under continuous and invasive monitoring, with epidural anesthesia uneventfully. In this context, this seems to be a safe strategy for such cases.

\section{References}

1. Trivedi RA, Kirkpatrick PJ - Arteriovenous malformations of the cerebral circulation that rupture in pregnancy. J Obstet Gynaecol. 2003;23(5):484-489.

2. Soderman M, Andersson T, Karlsson B et al. - Management of patients with brain arteriovenous malformations. Eur J Radiol. 2003;46:195-205.

3. Ogilvy CS, Stieg PE, Awad I et al. - Recommendations for the Management of Intracranial Arteriovenous Malformations: A Statement for Healthcare Professionals From a Special Writing Group of the Stroke Council, American Stroke Association. Stroke. 2001;32:1458-1471.
4. Friedlander RM - Arteriovenous Malformations of the Brain. $\mathrm{N}$ Engl J Med. 2007;356:2704-2712.

5. Di Rocco C, Tamburrini G, Rollo M - Cerebral arteriovenous malformations in children. Acta Neurochir. 2000;142:145-146.

6. Sinha PK, Neema PK, Rathold RC - Anesthesia of Intracranial Arteriovenous Malformations. Neurol India. 2004;52(2):163169.

7. Newfield P, Cottrell J - Handbook of Neuroanesthesia, 4th edition, Philadelphia, Lippincott Williams and Wilkins. 2007;278295.

8. Le LT, Wendling A - Anesthetic Management of Cesarean Section in a Patient With Rupture of a Cerebellar Arteriovenous Malformation. J Clin Anesth. 2009;21(2):143-148.

9. Horton JC, Chambers WA, Lyons SL et al. - Pregnancy and the risk of Haemorrhage from Cerebral Arteriovenous Malformations. Neurosurgery. 1990;27(6):867-872.

10. Coskun D, Mahli A, Yilmaz Z et al. - Anaesthetic management of caesarean section of a pregnant woman with cerebral arteriovenous malformation: a case report. Cases J. 2008;1:327329.

11. Lanzino G, Jensen ME, Cappelletto B et al. - Arteriovenous malformations that rupture during pregnancy: a management dilemma. Acta Neurochir. 1994;126:102-106.

12. Lynch J C, Andrade R, Pereira C - Hemorragia Intracraniana na gravidez e puerpério - Experiência com 15 anos. Arq Neuropsiquiatr. 2002;60(2A):264-268.

13. Robinson JL, Hall CS, Sedzimir CB - Arteriovenous Malformations, Aneurysms and Pregnancy. J Neurosurg. 1974;41:63-70.

14. Dias MS and Sekhar - Intracranial Haemorrhage from Aneurysms and Arteriovenous Malformations during Pregnancy and the Puerperium. Neurosurgery. 1990;27(855):444-446.

15. Ng K, Parsons J, Cyna AM et al. - Spinal versus epidural anaesthesia for caesarean section. Cochrane Database Syst Rev. 2004;(2):CD003765Cochrane.

16. Wong C - Spinal and Epidural Anesthesia, 1st Edition, Chicago, McGraw Hill, 2007;281-304.

17. Langesaeter E, Dyer RA - Maternal haemodynamic changes during spinal anaesthesia for caesarean section. Curr Opin Anesthesiol. 2011;24:242-248. 